\title{
The status and future of EUV astronomy
}

\author{
M. A. Barstow*, S. L. Casewell \\ Department of Physics and Astronomy, University of Leicester, University Road, \\ Leicester, LE1 7RH, UK \\ J. B. Holberg \\ Lunar and Planetary Laboratory, 1541 East University Boulevard, Sonett Space Sciences \\ Building, University of Arizona, Tucson, AZ 85721, USA \\ M.P. Kowalski \\ Naval Research Laboratory, 4555 Overlook Ave SW Washington, DC 20375, USA
}

\begin{abstract}
The Extreme Ultraviolet wavelength range was one of the final windows to be opened up to astronomy. Nevertheless, it provides very important diagnostic tools for a range of astronomical objects, although the opacity of the interstellar medium restricts the majority of observations to sources in our own galaxy. This review gives a historical overview of EUV astronomy, describes current instrumental capabilities and examines the prospects for future facilities on small and medium-class satellite platforms.
\end{abstract}

Keywords: Extreme Ultraviolet, Spectroscopy, White Dwarfs, Stellar Coronae, Interstellar Medium

\section{Introduction}

The Extreme Ultraviolet (EUV) nominally spans the wavelength range from 100 to $912 \AA$, although for practical purposes the edges are often somewhat indistinct as instrument band-passes may extend short-ward into the soft X-ray or long-ward into the far ultraviolet (far-UV). The production of EUV photons is primarily associated with the existence of hot, $10^{5}-10^{7} \mathrm{~K}$ gas

\footnotetext{
*Corresponding Author: mab@le.ac.uk
} 
in the Universe. Sources of EUV radiation can be divided into two main categories, those where the emission arises from recombination of ions and electrons in a hot, optically thin plasma, giving rise to emission line spectra, and objects which are seen by thermal emission from an optically thick medium, resulting in a strong continuum spectrum which may contain features arising from transitions between different energy levels or ionisation stages of several elements. Examples of the former category are single stars and binary systems containing active coronae, hot $\mathrm{O}$ and $\mathrm{B}$ stars with winds, supernova remnants and galaxy clusters. Hot white dwarfs, central stars of planetary nebulae $(\mathrm{CPN})$ and neutron stars are all possible continuum sources and belong to the latter category. Cataclysmic variable binaries, where material is being transferred from a normal main sequence star (usually a red dwarf) onto a white dwarf, may well contain regions of both optically thin and optically thick plasma. Perhaps the most unique contribution EUV observations can make to astrophysics in general, is by providing access to the most important spectroscopic features of helium - the He I and He II ground state continua together with the He I and He II resonance lines. These are the best diagnostics of helium, the second most cosmically abundant element. The line series limits are at $504 \AA$ and $228 \AA$ for He I and He II, respectively.

Since most elements have outer electron binding energies in the range 10$100 \mathrm{eV}$, corresponding closely to the energies of EUV photons, any EUV radiation will interact strongly in any material through which it passes, including the interstellar medium (ISM). Early measurements of the density of interstellar gas indicated that it would be completely opaque to any EUV radiation arising from outside the Solar System (Aller, 1959). This view remained unchallenged for more than a decade, but increased understanding of the ISM indicated a high degree of patchiness and significantly lower densities in some directions than first assumed. Coupled with a more sophisticated analysis of the absorption cross-sections across the EUV range by (Cruddace et al 1974, Figure 1), it became evident that EUV sources should be detectable out to distances of a few hundred parsecs and at the shortest wavelengths (bordering on the soft X-ray) some extragalactic observations are possible.

This review outlines the development of EUV astronomy during the past 40 years, covering the early experiments on sub-orbital missions, through the major satellite-borne survey missions, to the development of spectroscopic instruments. Much of this history has been comprehensively covered in Barstow \& Holberg (2003). Therefore, we summarise just the main ele- 
ments here, concentrating on the most recent developments and the prospects for new missions, not included in that book.

\section{Sounding rockets and early missions}

With the realisation that galactic sources of EUV radiation may be detectable from above the Earth's atmosphere, a number of investigators, mainly at the Space Sciences Laboratory of the University of California, Berkeley, embarked on a series of sounding rocket missions during the early 1970s. These experiments did not use imaging instruments, although flux concentrators were used, and had limited sensitivity. The sub-orbital nature of the programme also restricted the observing time to just a few minutes. While several thousand square degrees of the sky were surveyed, only a single source, most likely associated with the cataclysmic variable VW Hyi, was detected (Henry et al, 1976).

An opportunity to carry out a more extended search for EUV sources arose in 1975 through the Soviet-American Apollo Soyuz mission (officially termed the Apollo Soyuz Test Project, ASTP), a well publicised link up between US and Soviet astronauts in orbit, as a demonstration of international good will. A number of science instruments were allowed to piggyback on the Apollo capsule for the 9 day duration of the mission. Two EUV instruments were included in the programme, the Extreme Ultraviolet Telescope (EUVT, Bowyer et al 1977a) and the Interstellar Helium Glow Experiment (Bowyer et al, 1977b). More than 30 potential EUV sources were observed, with exposure times up to 20 minutes, yielding detections of four prototypical sources: two hot white dwarfs, HZ43 (Lampton et al, 1976) and Feige 24 (Margon et al, 1976), the cataclysmic variable SS Cyg (Margon et al, 1978) and the nearby flare star Proxima Centauri (Haisch et al, 1977). ASTP clearly demonstrated the feasibility of EUV astronomy and that new missions with greater sensitivity could explore the wavelength range with expectation of success.

\section{Sky surveys}

While flux concentrators (such as ASTP) could collect photons over a significantly large sky area, they were unable to discriminate between back-

ground and source photons. The development of true imaging systems through 


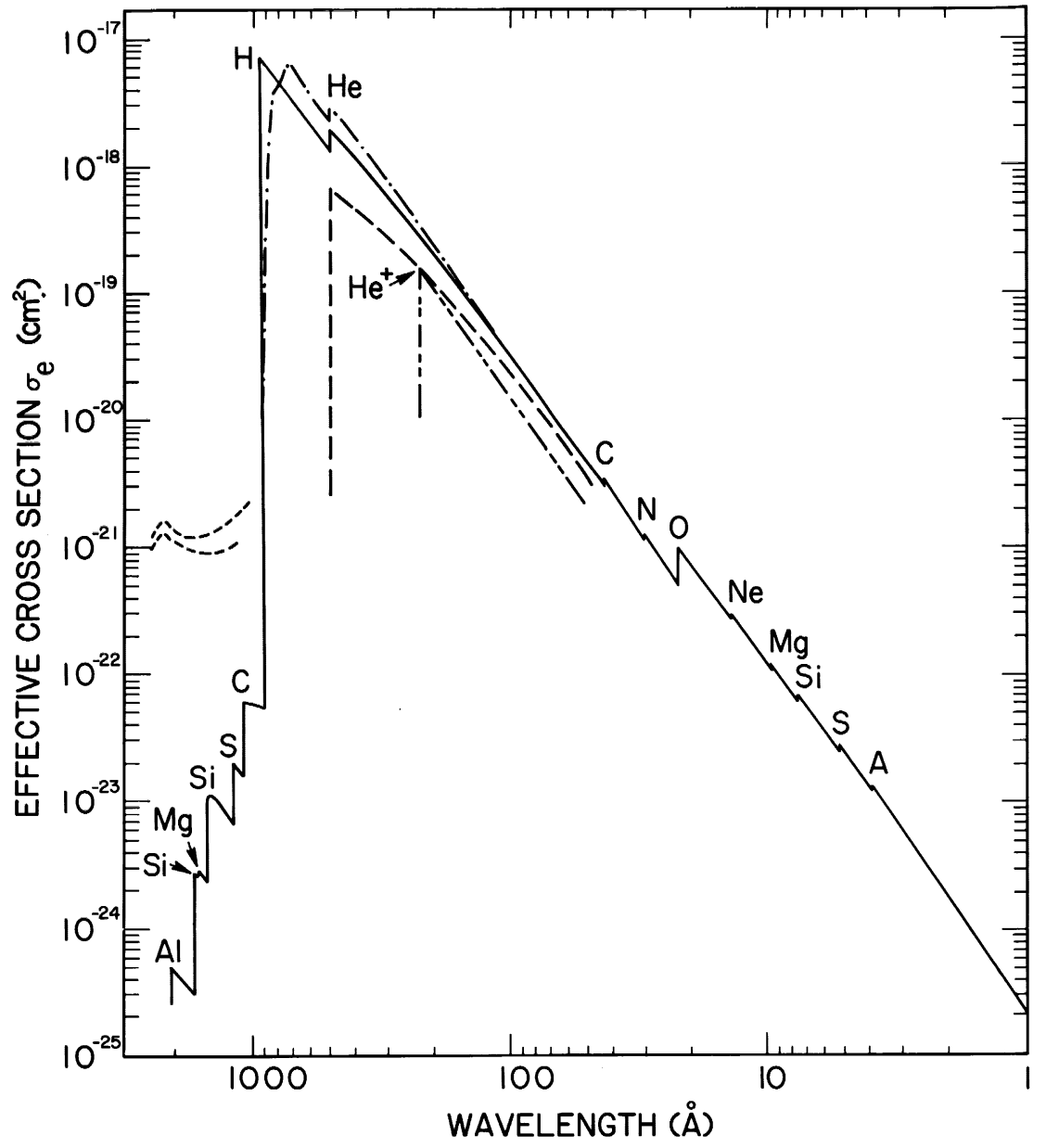

Figure 1: Effective cross-section of the interstellar medium. —— gaseous component with normal composition and temperature; -.- hydrogen, molecular form; - - H II region about a B star; - - - - H II region about an O star; - - - - dust (from Cruddace et al 1974.) 


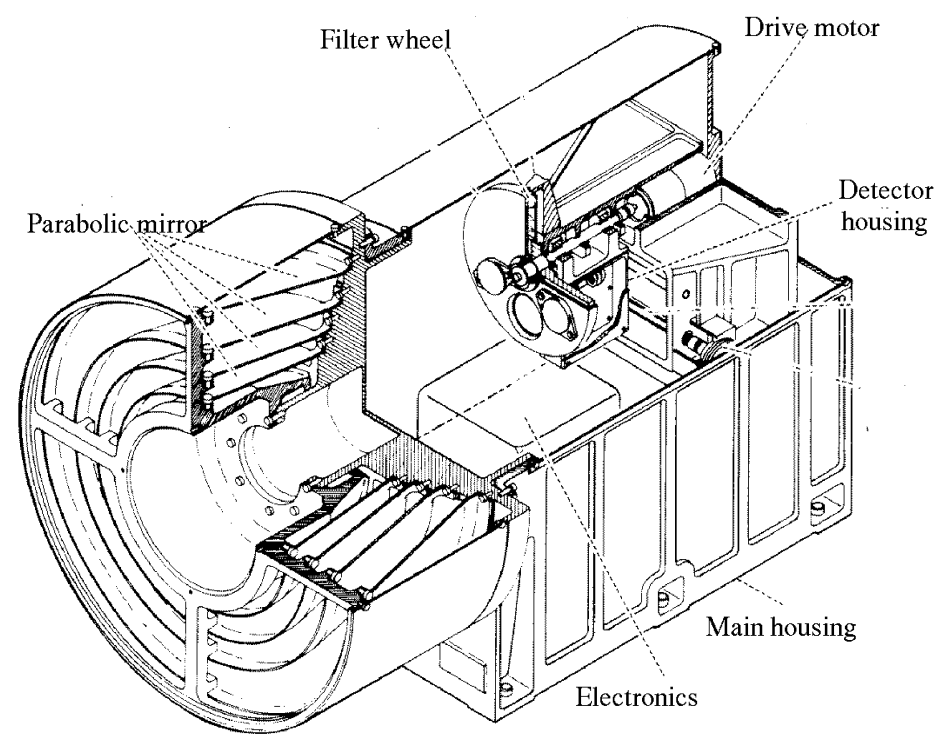

Figure 2: A schematic diagram of the EUV telescope flown on board the Apollo-Soyuz mission in 1975.

grazing incidence mirror technology in the late 1970s had an important impact on the sensitivity of X-ray and EUV instrumentation, since the background photons could be subtracted from the source image. Coinciding with these was the growing need to find out just what sources of cosmic EUV radiation were present to determine the scope and importance of EUV observations in astrophysics. Therefore, in common with all new energy windows in astronomy, all-sky surveys were planned for the EUV wavelength range. Two projects were adopted, the NASA Extreme Ultraviolet Explorer (EUVE) and the UK Wide Field Camera (WFC), to be flown as part of the German-led ROSAT X-ray sky survey mission.

These missions had similar objectives, to search the whole sky for EUV sources, but slightly different technical approaches. The WFC (Sims et al 1990, Figure 3) was a single telescope with 3 nested mirrors, designed to cover a wavelength range from 60 to $200 \AA$, at the short end of the EUV band, where the ISM is most transparent and most sources expect to be visible. However, the instrument also had a capability for observing longer wavelengths following the survey where the telescopes were pointed directly 


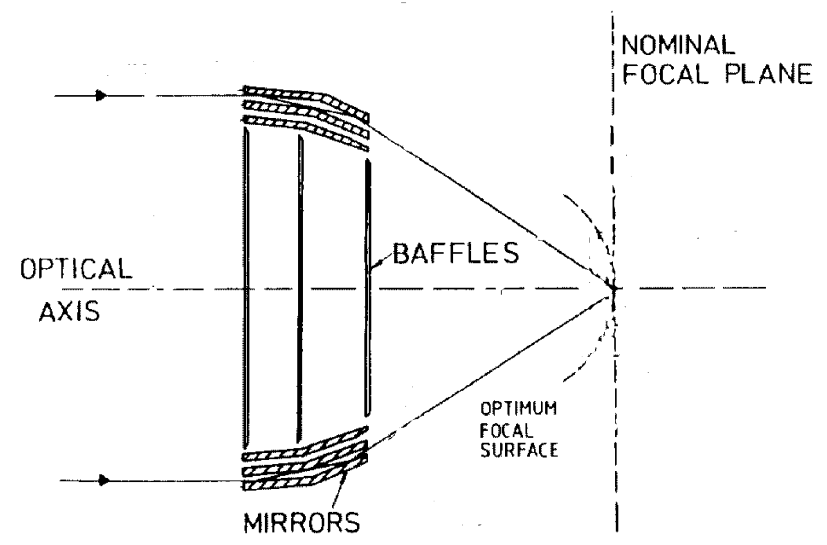

Figure 3: Schematic diagram of the optical systems of the ROSAT WFC.

at specific targets.

EUVE (Bowyer \& Malina (1991a,b); Figure 4, 5) had a complement of three single grazing incidence telescopes covering different wavelength ranges and providing all sky survey coverage extending to $800 \AA$. In addition, EUVE carried a spectrometer for pointed observations following the survey phase of the mission. We will discuss the spectrometer in the next section. However, half the effective area of the spectrometer was utilised for a deep survey, observing at $90^{\circ}$ to the main survey and giving exposure times significantly greater than the main sky survey but over a restricted region of the sky.

The scientific highlights of the ROSAT WFC and EUVE have been well documented in the scientific literature as well as by Bowyer, Drake \& Vennes (2000) and Barstow \& Holberg (2003). The principal outcome of the sky surveys was the detection of several hundred sources of EUV radiation. The WFC was launched in 1990 and carried out it survey between June 1990 and January 1991. A first release of source detections, the Bright Source Catalogue, included 383 objects (Pounds et al, 1993). This number was increased to 479 in a later release, the 2 RE catalogue (Pye et al, 1995), with improvements in attitude reconstruction, background screening and source detection methods. EUVE was launched in June 1992 and, consequently, carried out its sky survey approximately 2 years after the WFC. A preliminary bright source list was published by Malina et al (1994) and followed by the first 


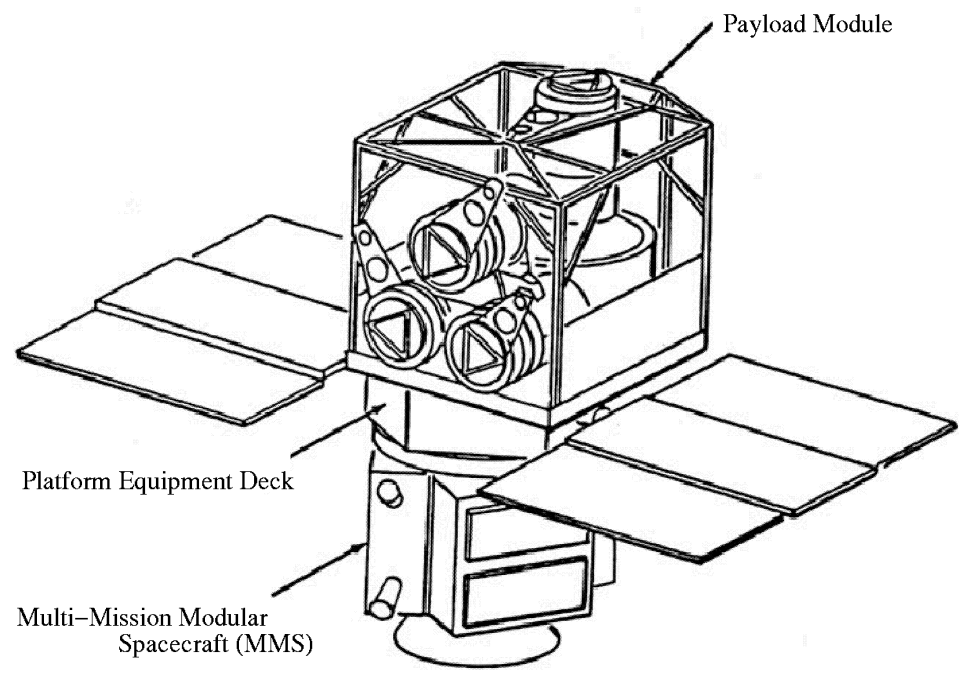

Figure 4: Schematic diagram of the EUVE payload, showing the all-sky survey scanners and the deep survey/spectrometer telescope pointing at right-angles to these.

EUVE catalogue of 410 sources (Bowyer et al, 1994) comprising 287 objects from the all-sky survey and 35 from the deep survey. A further 88 objects were drawn from the bright source list identified through a variety of special processing techniques. Following the main survey, during spectroscopic follow-up observations, the sky survey telescopes continued to observe field in the so-called right angle programme. The second EUVE source catalogue (Bowyer et al, 1996) included objects from this partial, deep survey of the sky as well as the re-processed all-sky and deep surveys yielding a total of 734 sources.

Despite the wide range of sources that emit in the EUV, the vast majority of objects detected fall in to two categories, hot white dwarfs and stellar coronal sources. These are seen in roughly equal numbers, but with about $20 \%$ more coronal sources. In contrast, there are only relatively small numbers of cataclysmic variables and $\mathrm{OB}$ stars. It is also evident that there are windows in the ISM through which it is possible to see out of the Galaxy, through the detection of about 2 extragalactic sources. It is not surprising that most of these are located at high galactic latitude where the ISM column densities are lowest. 


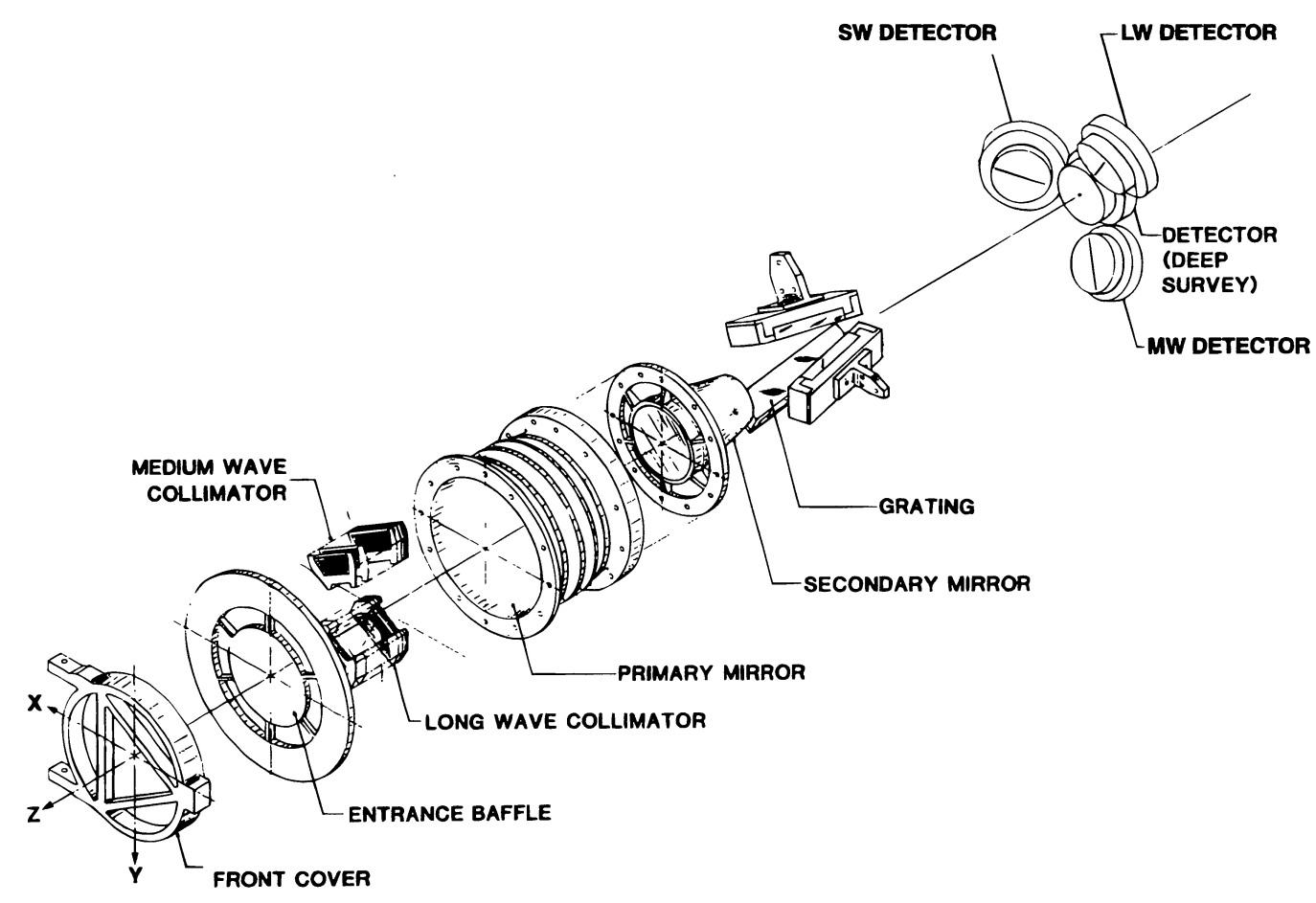

Figure 5: Exploded view of the EUVE Deep Survey/Spectrometer telescope. The axes drawn at the front of the instrument represent the DSS coordinate system. 
The major survey results concern the white dwarf and coronal populations. The photometric luminosities of the hottest white dwarfs are seen to be suppressed when compared with pure $\mathrm{H}$ model atmospheres, implying that their atmospheres contain significant quantities of heavier elements, identified from follow-up UV spectroscopy as principally, C, N, O, Si, Fe and $\mathrm{Ni}$ (see Barstow \& Holberg 2003). It should be mentioned that the ability to detect strong EUV continua from white dwarfs with effective temperatures as low as $\sim 2 \times 10^{4} \mathrm{~K}$ is a consequence of the low opacity of $\mathrm{H}$ in the EUV which allows the emergence of EUV fluxes from deeper, hotter layers. The coronal sources tend to lie much closer to the solar system, mostly within 50-60 pc, indicative of their lower luminosity, while white dwarf detections extend as far as 200 pc in significant numbers. An important subgroup of objects revealed by the surveys are a number of hot white dwarfs in relatively close binary systems, where, at visible wavelengths, the low luminosity white dwarf is hidden in the glare of its much brighter companion. In the EUV, the situation is reversed due to the high EUV luminosity of white dwarfs compared to coronal sources. These binaries are analogous to the Sirius system, which is only resolved into two components because of its relatively close proximity to Earth.

For completeness, it necessary to mention a third sky survey with the ALEXIS satellite (Priedhorsky et al, 1988). ALEXIS made use of normal incidence mirrors with multi-layer coatings, which, in principle, provide greater effective area than grazing incidence optics for the same aperture. However, the multilayer reflectivity has a narrow response spanning a few tens of $\AA$, which can limit the overall photon flux, particularly for broad-band continuum sources such as white dwarfs. Unfortunately, after launch one of the solar panels deployed prematurely. Although the instruments eventually became operational, limited attitude control and reconstruction made it difficult to analyse and understand the data collected. More than 18 steady EUV sources were detected but only one that was not already discovered by the WFC or EUVE. However, one of the ALEXIS science goals was detection of bright transients. Five were reported during the mission lifetime of which 3 were associated with known cataclysmic variables.

\section{EUV spectroscopy}

Photometric all-sky surveys are the fundamental sources of important information regarding the general properties of groups of objects in the EUV source population. However, when considering individual objects in detail, 
the amount of information that can be extracted from a few photometric data points is somewhat limited. Therefore, the ability to obtain spectroscopic follow-up observations is an important next step in the exploitation of any observational window. The inclusion of a spectrograph on the EUVE mission was an important addition, since it provided immediate access to spectroscopic follow-up data on completion of the all-sky survey. The instrument made use of the deep survey telescope placing three reflection gratings in the optical path, each occupying about $1 / 6$ of the collecting area, covering a total wavelength range from 70 to $760 \AA$ in three segments: SW,

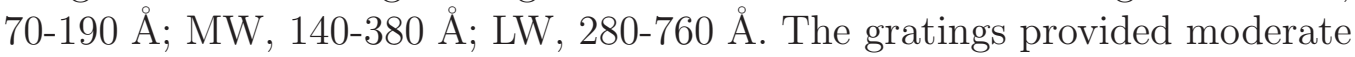
resolving power $(\sim 200)$ across the band, with typical average values of 0.5 $\AA, 1.0 \AA$ and $2.0 \AA$ for SW, MW and LW respectively.

With an effective area ranging from $\sim 0.2$ to $2 \mathrm{~cm}^{2}$, the EUVE spectrographs were restricted mainly to observing the brightest EUV sources. Even then, long exposure times were typically required from several thousand to several tens of thousands of seconds. Nevertheless, many sources were observed and some important and powerful diagnostic measurements obtained. There is insufficient space in this review to give a full picture of the work carried out, but we show a few examples of EUVE spectra and some of the key highlights. The types of object observed divide simply into continuum and line emission sources. The former are mostly white dwarfs but include a number of cataclysmic variables and the $\mathrm{B}$ stars $\beta$ and $\epsilon$ CMa. The emission line sources are all stellar coronae. 


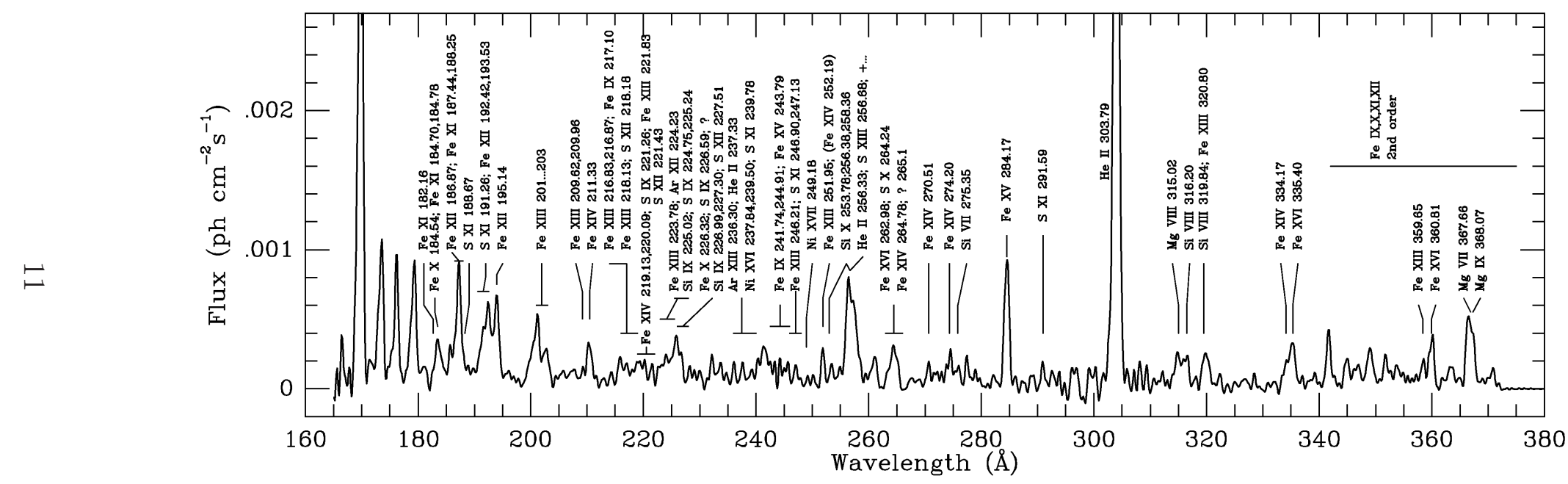

Figure 6: Flux calibrated MW EUV spectrum of Procyon. Individual lines and unresolved groups used in the analysis of Drake et al (1995) are labelled, together with a number of other features not used. The line identifier '2nd' refers to shorter wavelength lines seen in second order. 


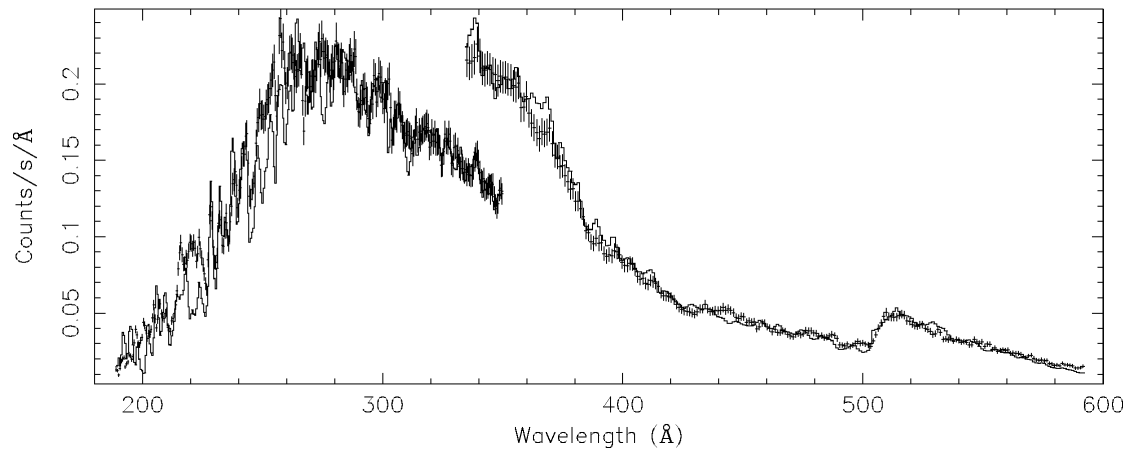

Figure 7: EUVE count spectrum of G191-B2B covering the wavelength range 180-600^. The data points (error bars) are compared with the predictions of a non-LTE model atmosphere calculation including the effects of interstellar absorption ( $\mathrm{H}$, He I and He I column densities are $2.1 \times 10^{18}, 1.8 \times 10^{17}$ and $7.9 \times 10^{17} \mathrm{~cm}^{-2}$ respectively). The discontinuity near $320 \AA$ arises from differing spectrometer effective areas for which these data are not corrected.

Figure 6 shows a section of one of the best cool star spectra obtained by EUVE, of Procyon, illustrating the range of elements and ionisation stages that can be observed (Drake et al, 1995). In a spectrum like this there will be lines that are sensitive to the plasma temperature and others that are density sensitive diagnostics. Combining this information allows a determination of the physical properties of the coronal gas, including the emission measure distribution and elemental abundances.

In the continuum sources such as white dwarfs, spectral features are typically seen in absorption. In the hot, dense atmospheres there can be a very large number of high excitation features that cannot be resolved and appear as blends. A good example is the H-rich DA white dwarf G191-B2B (Figure 7). The short wavelength flux is strongly suppressed by the opacity of heavy elements in the photosphere and there are many broad absorption features, which are blends of many individual lines. Initially, for stars like G191-B2B, the agreement between the data and stellar model atmospheres was very poor. It was realised (Lanz, et al, 1996) that it was necessary to include many more atomic transitions in the models to take account of the true opacity in the stellar atmospheres. However, even though the general shape of the spectra was reproduced, there was detailed disagreement when modelling the individual features. In addition, to model the shorter wavelength 
flux level, below $200 \AA$, required development of stratified atmospheres where the abundance of Fe in particular was allowed to vary with depth (Barstow, \& Hubeny, 1998).

As outlined in the introduction, the EUV spectral range is unique in containing the He I and He II resonance lines. At the resolution of EUVE the individual lines in the series are not generally resolved but are evident in a step function absorption "edge" generated by the blending of the lines near the series limit. This can be seen in Figure 7, which show the He I interstellar absorption edge at $504 \AA$. In this example, the signature of the He II edge at $228 \AA$ and a He I autoionisation feature at $206 \AA$ are blended with the photospheric absorption lines and hard to detect. However, when the white dwarf atmosphere has fewer metals, these features can be seen very clearly and provide powerful diagnostics on the physical state of the local ISM (Figure 8). In particular, the fractional ionisation of $\mathrm{H}$ and $\mathrm{He}$ can be determined and mapped across the local ISM. Interestingly, within the observational errors, the $\mathrm{H}$ and $\mathrm{He}$ ionisation fractions appear to be quite uniform ( $23 \%$ and $35 \%$ respectively) in space (Barstow et al, 1997). This has been interpreted as ionisation of the local interstellar cloud surrounding the solar system by a past supernova explosion followed by a period of recombination (Jenkins , 2013). The current ionisation fractions indicate an elapsed time of 2.1-3.4 million years since the onset of recombination.

\section{High resolution spectroscopy with J-PEX}

The EUVE spectrometer made a number of very important advances in our understanding of compact objects, coronal sources and the interstellar medium through observations of several dozen of the brightest sources in the EUV sky. Nevertheless, the limited effective area and spectral resolution available placed considerable restrictions on the physical information that

could be extracted from the data. Particular issues arise in the presence of large numbers of closely spaced spectral features that were unresolved. In addition, limited dynamical information could be obtained from EUVE data, since typical gas or spatial velocities were much smaller than the resolving power. Therefore, it was inevitable that the science drivers following EUVE would emphasise much improved spectral resolution and telescope collecting area.

It is well understood that the grazing incidence optical systems used by the WFC and EUVE are relatively inefficient light collectors, with large 


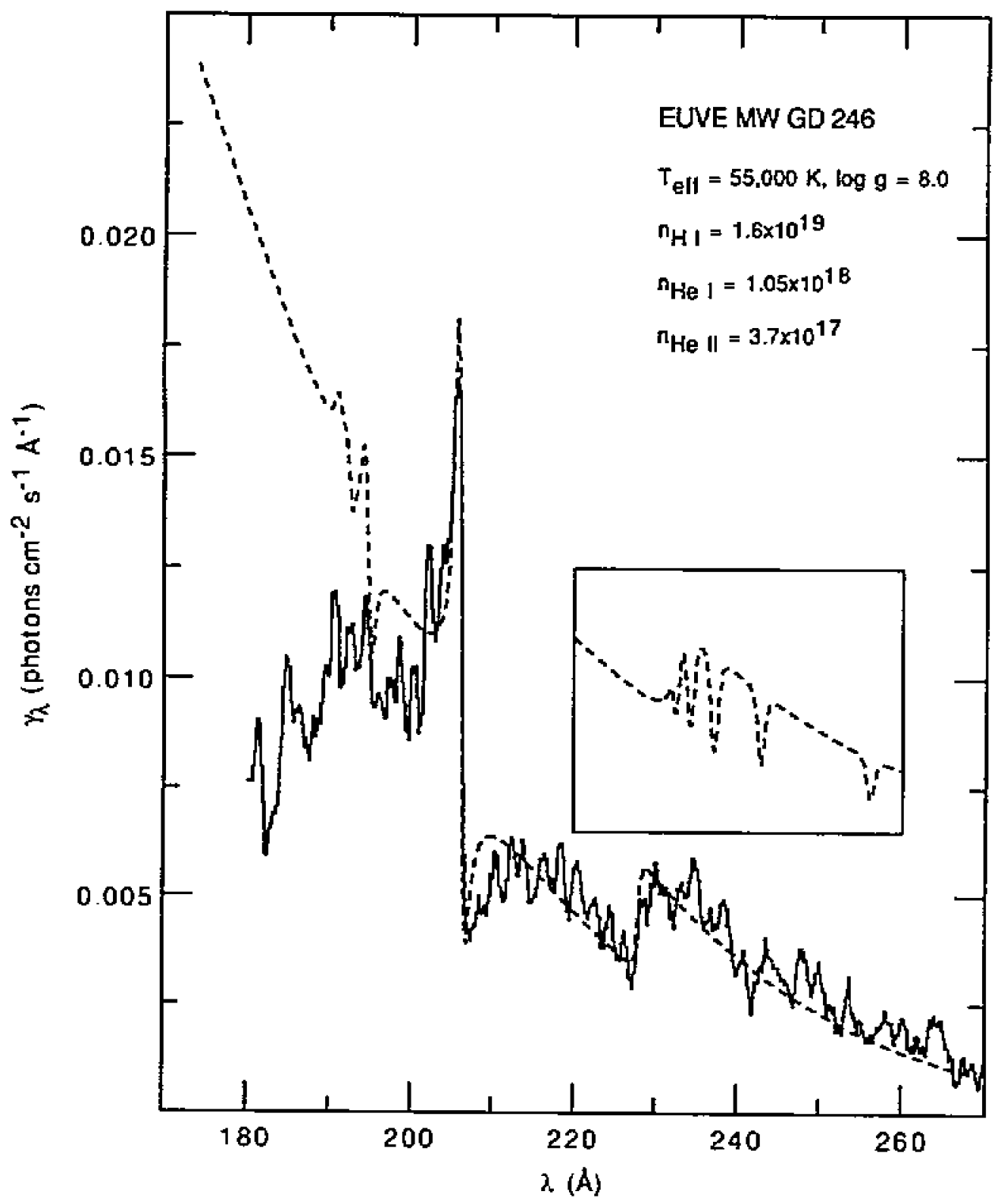

Figure 8: Observation of the DA white dwarf GD246 showing the region of the spectrum between 180 and $270 \AA$ compared to a pure $\mathrm{H}$ atmosphere model including the interstellar opacity from $\mathrm{H}$ and He, showing the He I autoionisation feature at $206 \AA$ and the He II absorption edge at $228 \AA$ (from Vennes et al 1993 . 

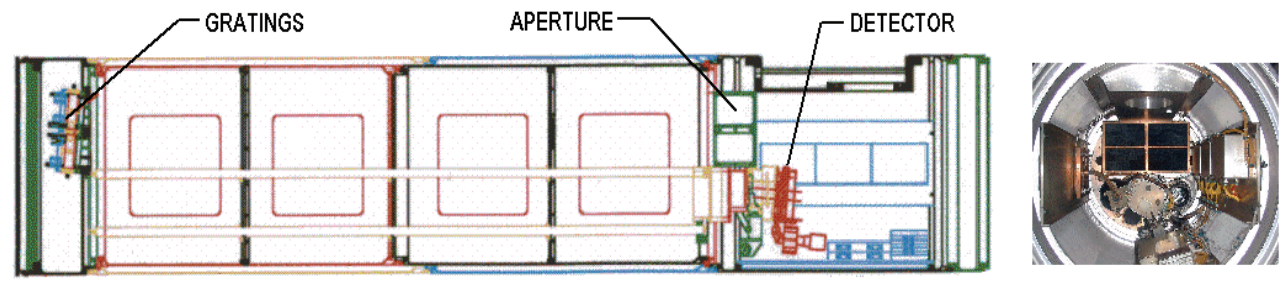

Figure 9: The J-PEX high-resolution spectrometer payload, designed and constructed for launch by a Terrier boosted Black Brant IX sounding rocket. The J-PEX spectrometer has made two successful flights, observing the isolated DA white dwarf G191-B2B and the DA+dM binary Feige 24, with the aim of examining their photospheric composition and searching for the presence of photospheric and interstellar He II. With a spectral resolution of 4000-6000 (depending on the flight configuration) it is possible to achieve the goal of developing the instrument, to resolve the individual lines of the He II Lyman series and separate them from other features from heavier elements.

collecting areas leading to large masses for the optics. The ALEXIS mission pointed the way forward by producing lightweight telescopes based on multilayer-coated normal incidence mirrors. However, these only provided narrow band photometry. The Joint astrophysical Plasmadynamic Experiment (J-PEX) is a sounding rocket-borne spectrometer, constructed by the Naval Research Laboratory, the University of Leicester and the Mullard Space Science Laboratory, delivering both high throughput and spectroscopy. The instrument is a slitless design, which employs a figured spherical grating in a Wadsworth mount. This provides focusing and dispersion in a single optical element, keeping reflectivity losses to a minimum. As a result of practical upper limits on the grating size at the time the payload was constructed, four individual identical grating segments were utilised to maximise the geometric collecting area. The design of the J PEX payload is shown in Figure 9. The EUV light from the star enters a collimator, which minimises the EUV background flux into the spectrometer, and strikes the grating at an angle of incidence of $4.85 \mathrm{deg}$. The grating is coated with a multilayer designed for high efficiency in the band 220-245 $\AA$. The diffracted radiation is focused onto an MCP detector mounted on the grating optical axis, a configuration, which minimises aberrations. Diffracted and scattered EUV/FUV background is trapped by baffles (not shown) within the spectrometer, while residual background reaching the detector is attenuated by an aluminium filter. The instrument has been described in detail by (Cruddace et al, 2002) 
and (Barstow et al, 2005).

Figures 10 and 11 show the J-PEX spectrum of G191-B2B. Cruddace et al (2002) provided an initial analysis of this data and Barstow et al (2005) carried out more detailed follow-up work. We summarise the main results of Barstow et al (2005) here who studied both homogeneous and stratified mixtures of photospheric material. In contrast to earlier work, based on EUVE spectra Barstow, \& Hubeny (1998), the J-PEX data are best matched by the homogeneous models. This is illustrated in Figures 10 and 11, which compare the best fit between the two types of stellar model atmospheres. Particularly good agreement is obtained in the $\sim 228 \AA$ to $230 \AA$ wavelength range. Apart from the HeII Lyman series, no strong absorption lines are predicted by the models in this range. We can clearly identify $\lambda 228.54$ $\AA$ (left hand arrow in Fig. 10), and blends of He lines down to the series limit (marked by the bracket in Fig. 10). The apparent emission feature at $227.9 \AA$ is an artifact of the effect of He II line series opacity on the stellar emission, which was predicted by modelling before the J-PEX flight. The absorption line at $229.0 \AA$ coincides with predicted He II and O III features and is probably a blend of both (right hand arrow in Fig. 10).

All EUV studies of G191-B2B have required an interstellar component (or components) of HeII opacity to explain the observed spectra. At the resolution of EUVE and in the presence of absorption from many other species, this material could not be directly detected. However, the J-PEX data clearly reveal the interstellar HeII Lyman series lines, and further they imply that there is a contribution from photospheric He, although the latter is only formally an upper limit, as the predicted strength of the $243 \AA$ line is similar to the noise in the spectrum (see Fig 11) and zero photospheric He is not a significantly different solution.

The DA+dM binary Feige 24 was observed as a comparison with G191B2B. The two white dwarfs have very similar temperatures and compositions, as measured by UV spectroscopy. However, Feige 24 is a member of a close, $4.25 \mathrm{~d}$ period, binary system with its $\mathrm{dM}$ companion and will have passed through a common envelope phase of evolution, which may have affected the structure of the white dwarf. If this had happened, significantly reducing the mass of the $\mathrm{H}$ envelope, this would be revealed through detection of photospheric He in the EUV. Figure 12 shows the spectrum recorded by J-PEX compared to stellar model atmospheres with varying $\mathrm{H}$ envelopes. The very surprising result of this work is that there is no evidence for the existence of a thin H-layer or any photospheric He. This is illustrated in 
a)

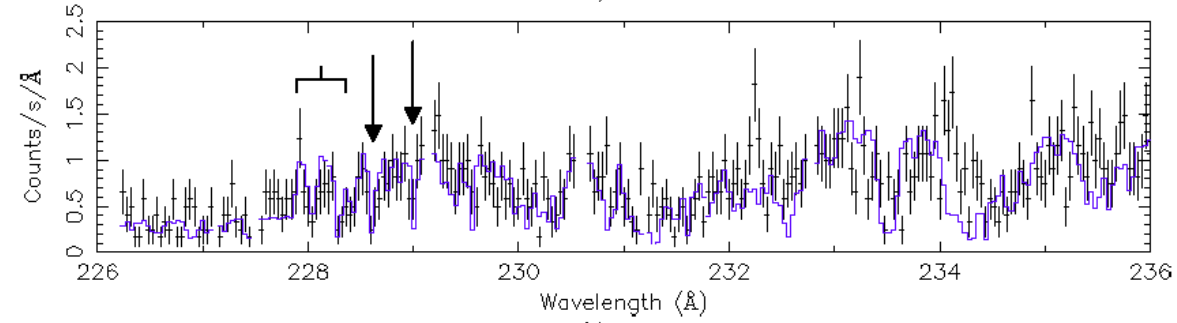

b)

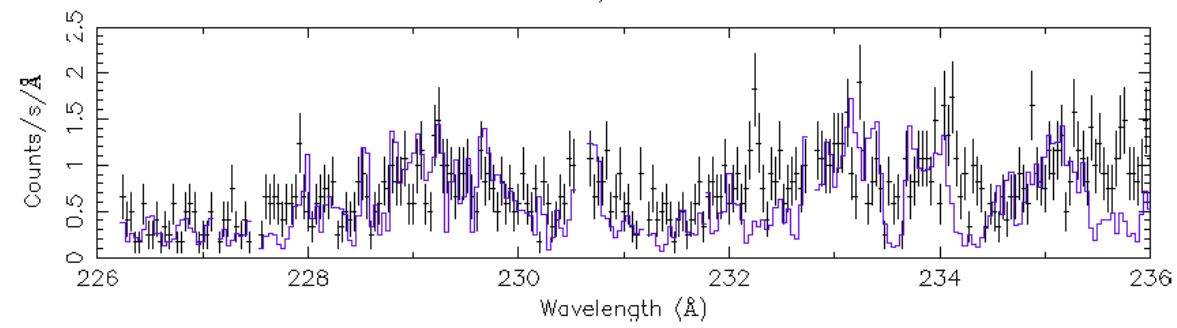

Figure 10: Comparison of the best-fit spectral models for each atmosphere type (histograms) with the $226 \AA$ to $236 \AA$ region of the J-PEX spectrum of G191-B2B (error bars). a) Homogeneous mixture of all elements, b) Self-consistent radiative levitation/diffusion PRO2 model. In a) the bracket identifies the converging He II Lyman series lines and the series limit, the left arrow marks HeII $228.54 \AA$ and the right arrow a blended feature of O III and He II at 229.0 А. 
a)

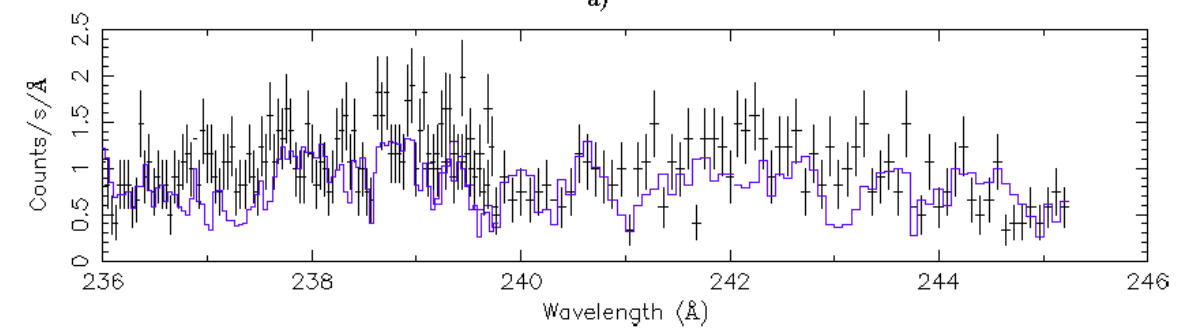

b)

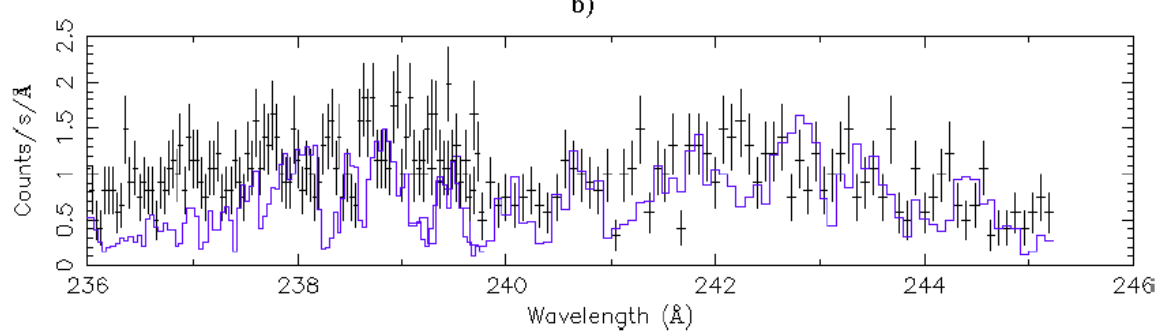

Figure 11: Comparison of the best-fit spectral models for each atmosphere type (histograms) with the $236 \AA$ to $246 \AA$ region of the J-PEX spectrum of G191-B2B (error bars). From the top a) Homogeneous mixture of all elements, b) Self-consistent radiative levitation/diffusion PRO2 model. 


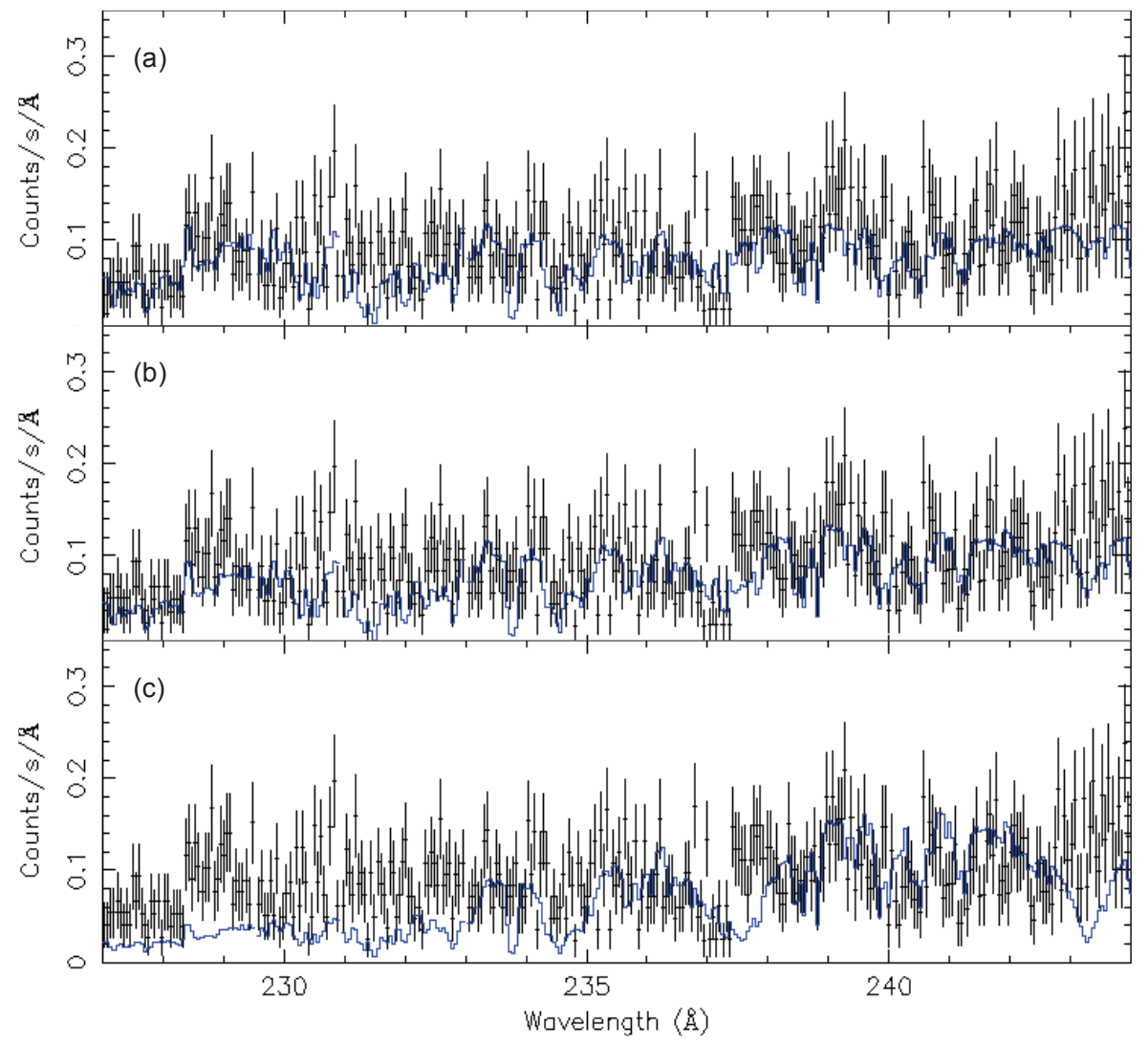

Figure 12: J-PEX high resolution spectrum of Feige 24 (black error bars) compared to a composite photospheric and ISM model spectrum (blue histogram). (a) Best fit model with H-layer mass of $1.2 \times 10^{-13} \mathrm{M}_{\odot}$, (b) H-layer mass $=3 \times 10^{-14} \mathrm{M}_{\odot}$, and c) H-layer mass $=10^{-14} \mathrm{M}_{\odot}$. 
Figure 12(a), where the H-layer mass has converged to the upper limit of the grid $\left(1.2 \times 10^{-13} \mathrm{M}_{\odot}\right)$, a level at which no He is detectable. Our sensitivity to the H-layer mass is illustrated in Figures $12(\mathrm{~b})$ and $12(\mathrm{c})$, which show the effect of decreasing the H-layer mass in the model to $3 \times 10^{-14}$ and $10^{-14} \mathrm{M}_{\odot}$, respectively. The strong constraints on the amount of He present are provided by the absence of detectable He II lines, particularly at 237.3 $\AA$ and $243 \AA$, and the predicted suppression of the continuum flux below $\sim$ $233 \AA$ for thinner H-layers.

\section{Potential future missions: APEX and SIRIUS}

The J-PEX spectrograph has demonstrated the power of high-resolution spectroscopy. Its high effective area provided a capability that could obtain data of reasonable signal-to-noise in the few hundred seconds exposure available in a sounding rocket flight. Nevertheless, the number of targets that are bright enough for such observations is limited. Furthermore, to observe a reasonable sample of objects would require an unrealistic number of rocket flights. Hence, the J-PEX technology and the science it enables can only be fully exploited if it is flown on a long duration satellite platform. The J-PEX gratings were tuned to cover the wavelength range 220-260 $\AA$, addressing the science goals of studying the He II Lyman line series and heavy elements in white dwarf spectra. The overall spectral coverage is limited by the multilayer coating, where there is a tradeoff between reflectivity and wavelength coverage. Maximising reflectivity reduces the bandpass. J-PEX was an optimised solution to match the required wavelength range for the science goals. Further important white dwarf and interstellar medium science could be obtained over a wider wavelength range. In addition, extending to shorter wavelengths would enable crucial observations of coronal sources and cataclysmic variable stars, which were too faint for J-PEX.

As a sounding rocket payload, J-PEX is a relatively low cost instrument with proven technology at a high level of technological readiness. No further technical development is necessary for translation to a satellite mission. Therefore, it is also an excellent prospect for low cost satellite development if coupled with an appropriate platform and launch opportunity. The telescope has a $2 \mathrm{~m}$ long focal length enclosed in a $\sim 56 \mathrm{~cm}$ diameter cylindrical housing, which is small compared to typical large space observatories such as Chandra, XMM-Newton or HST. There are two main approaches to a satellite version of the spectrograph that have been investigated to date. One is 


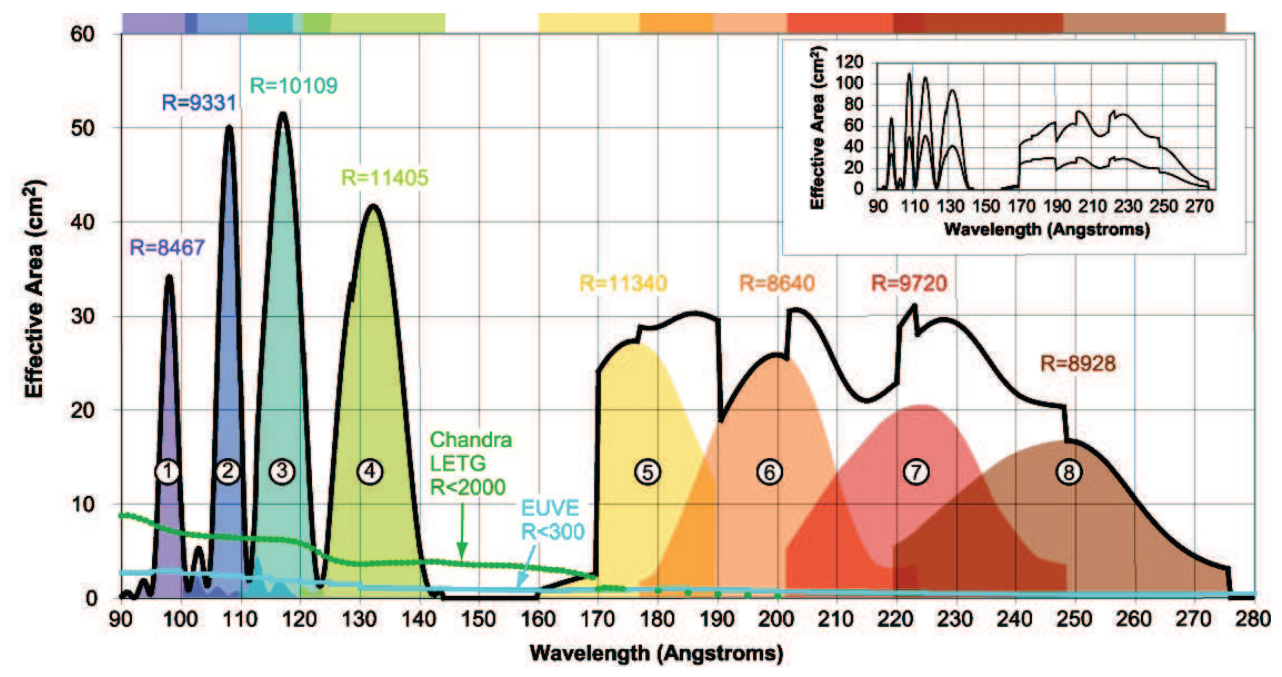

Figure 13: Baseline effective area of SAGE (inset: Baseline and Best-case effective area sums). The black curve is the cumulative effective area if the individual spectra are coadded.

to provide broad wavelength coverage by multiplexing several J-PEX style telescopes, each tuned to a different waveband, on a single platform. Such a package fits comfortably in to the usual physical and cost envelope of a small to medium mission opportunity (depending upon the agency). A recent example was the development of the SAGE concept in response to the recent ESA medium mission opportunities. This utilises eight telescopes of $3 \mathrm{~m}$ focal length, giving improved spectral resolution over J-PEX (see Barstow et al 2009). Figure 13 shows the effective areas of each telescope as a function of wavelength for a nominal payload that can study stellar coronae, white dwarfs and the local interstellar medium compared to EUVE and the Chandra LETG. The dramatic increase in the performance is evident. While the LETG has a good spectral resolution it is not sufficient for the study of the dynamics of stellar coronae, where it is necessary to resolve velocities of a few tens of $\mathrm{km} / \mathrm{s}$.

An alternative approach is make use of the modular nature of the original J-PEX design, which had four identical gratings to deliver the total required collecting area because it was not technically feasible to produce a large enough monolithic grating at the time. In J-PEX the grating multilayers were tuned to deliver the same wavelength coverage. The four spectra were 
SIRIUS High Resolution Spectrometer

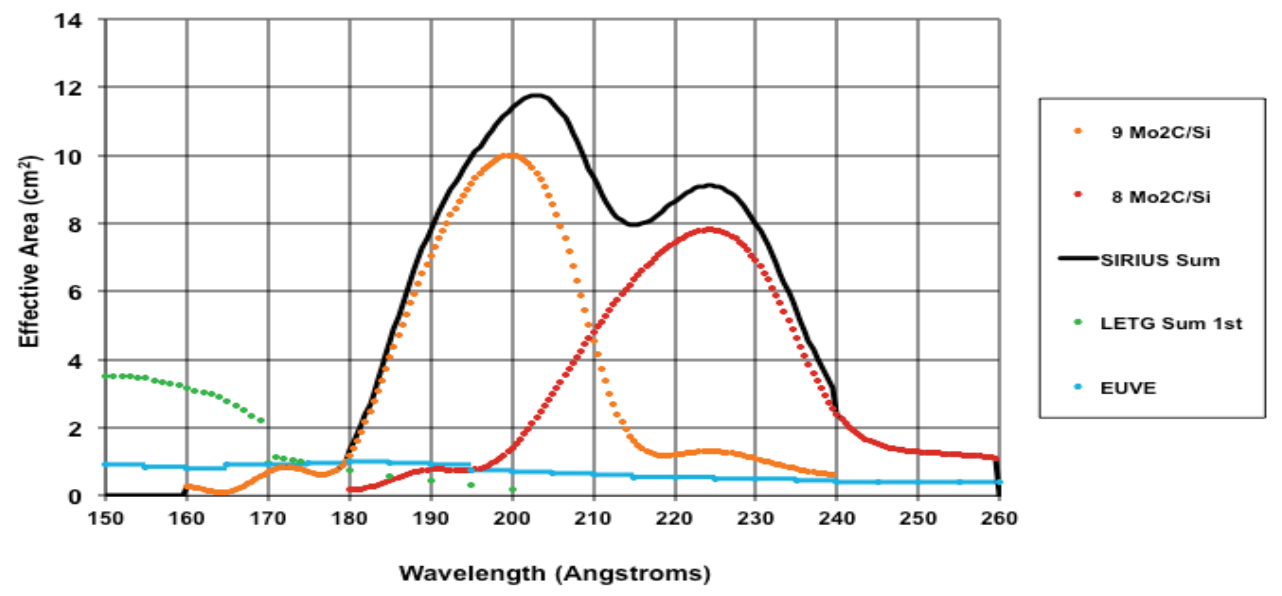

Figure 14: The effective area of SIRIUS, showing the individual wavebands, with Mo2C/Si coatings (orange and red), and the sum (black). Chandra LETG (green) and EUVE (blue) are shown for comparison.

imaged individually and then coadded during data analysis to achieve the full signal-to-noise available. However, with the longer exposures available from a satellite platform, the four gratings could, in principle, be tuned to different wavelength ranges to give broad coverage. This approach was applied in the SIRIUS proposal in response to the 2012 ESA small (S) mission call (Barstow et al, 2012). The four gratings were divided into two pairs, each pair tuned to cover the wavelength range 17-260 $\AA$ (Figure 14). Although this yields reduced effective area and spectral coverage when compare to SAGE, much of the science is retained, allowing the major goals of coronal, white dwarf and ISM studies to be achieved. Studies of cataclysmic variables and extragalactic sources requiring even shorter wavelength capabilities are sacrificed along the some of the shorter timescales of variability studies. However, the SIRIUS payload can be accommodated on a low cost satellite platform lower the mission cost to $\sim 50 \mathrm{M}$ Euros, a factor of 5-6 lower than a typical medium scale mission envelope.

The ability to provide a high resolution, high throughput EUV spectrometer as part of a low-cost mission arises not just from the extraordinary capability of the normal incidence grating technology but also from the avail- 

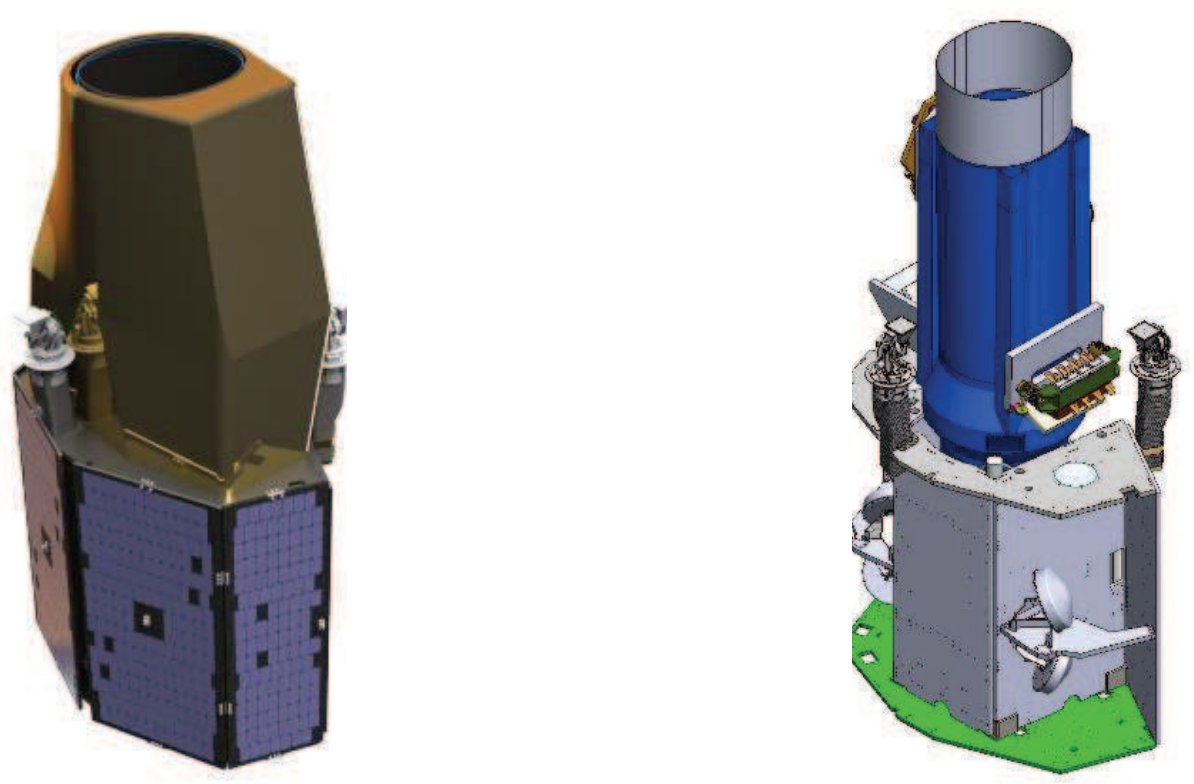

Figure 15: (left) The fully assembled SIRIUS satellite showing the spectrograph installed on the SSTL 300 spacecraft. (right) Cutaway, showing the telescope tube and spacecraft structure.

ability of high performance, low cost spacecraft solutions such as the SSTL 300 platform that underpinned the SIRIUS proposal. The spacecraft consists of the payload (SIRIUS instrument) mounted in the aperture of the SSTL 300 spacecraft (Figure 15). The platform design draws on extensive heritage from previous missions, using a standard electrical architecture for subsystems and interfaces.

\section{Conclusion}

Although early assumptions about the opacity of the ISM consigned the EUV to be a wavelength range where few if any objects could be studied outside the solar system, observations at EUV wavelengths have been shown to be of great important to astrophysics over the past $\sim 40$ years development of instrumentation to exploit this window in the electromagnetic spectrum. Sky surveys have revealed more than 700 sources of EUV radiation. However, interstellar opacity remains a dominant factor, as that vast majority of sources are galactic in nature. Therefore, to a great extent EUV astronomy will always be a niche for stellar astronomy and studies of the ISM. This presents 
a significant hurdle in developing the next generation high-resolution spectroscopy needed to further advance these subjects. An extremely capable, observatory-class telescope such as SAGE can be accommodated well within a medium class mission package. However, such a mission is often competing with other proposals that address the current priority areas of research such as cosmological studies or observations of exoplanets. Therefore, it is likely that the most viable future opportunity for EUV astronomy will be in the small mission arena, with a single telescope flying on a low cost platform, such as the SIRIUS concept.

\section{References}

Aller, L.H., Some aspects of ultraviolet satellite spectroscopy Publ. Ast. Soc. Pac., 71, 324-329, 1959.

Barstow, M.A., et al., Observatory class science with a low-cost EUV astronomy mission SPIE, 8443, 844303-844303-9, 2012.

Barstow, M.A., et al., Stellar and galactic environment survey (SAGE) ExA, 23, 169-191, 2009.

Barstow, M.A., et al., High-resolution extreme ultraiolet spectroscopy of G191-B2B: structure of the stellar photosphere and the surrounding interstellar medium MNRAS, 362, 1273-1278, 2005.

Barstow, M.A. \& Holberg, J.B., Extreme ultraviolet astronomy Cambridge University Press (Cambridge, UK, 2003).

Barstow, M.A. \& Hubeny, I., An alternative explanation of the EUV spectrum of the white dwarf G191-B2B invoking a stratified $\mathrm{H}+\mathrm{He}$ envelope including heavier elements, MNRAS, 299, 379-388, 1998.

Barstow, M.A., et al., Interstellar and photospheric opacity from EUV spectroscopy of DA white dwarfs MNRAS, 286, 58-76, 1997.

Bowyer, C.S., Drake, J.J. \& Vennes. S., Extreme Ultraviolet Astronomy Annual Reviews of Astronomy and Astrophysics, 38, 231, 2000.

Bowyer, S., The second extreme ultraviolet explorer source catalog ApJS, 102, 129-160, 1996. 
Bowyer, S., The first extreme ultraviolet explorer source catalog ApJS, 93, 569-587, 1994.

Bowyer, S \& Malina, R.F., The extreme ultraviolet explorer mission AdSpR, 11, 205-215, 1991a.

Bowyer, S., \& Mailina, R.F., The extreme ultraviolet explorer mission Extreme Ultraviolet Astronomy, Eds R.F. Malina \& S. Bowyer, (Pergamon press, New York, 1991b).

Bowyer, C.S., et al., Extreme ultraviolet survey: Experiment MA-083 aspo.nasa, 1, 49-70, 1977a.

Bowyer, C.S., et al., Interstellar helium glow: Experiment MA-088 aspo.nasa, 1, 71-83, 1977b.

Cruddace, R. G., et al., High resolution spectroscopy of G191-B2B in the extreme-ultraviolet ApJ, 565, L47-L50, 2002.

Cruddace, R., et al., On the opacity of the interstellar medium to ultrasoft X-rays and extreme-ultraviolet radiation, ApJ, 187, 497-504, 1974.

Drake, J.J., et al., The elemental composition of the corona of Procyon: Evidence for the absence of the FIP effect, Science, 267, 1470-1473, 1995.

Haisch, B.M., et al Extreme-ultraviolet observations of a flare on Proxima Centauri and implications concerning flare-star scale theory ApJ, 213, L119-L123, 1977.

Henry, P., et al., Detection of an extreme-ultraviolet source in the southern sky ApJ, 209, L29-L33, 1976.

Jenkins, E.B., The fractional ionization of the warm neutral interstellar medium ApJ, 764, 25-54, 2013.

Lampton, M., et al., Discovery of a non solar extreme-ultraviolet source ApJ, 203, L71-L74, 1976.

Lanz, T., et al., A self-consistent optical, ultraviolet and extreme-ultraviolet model for the spectrum of the hot white dwarf G191-B2B ApJ, 473, 10891093, 1996. 
Lyu, C-H., \& Bruhweiler, F.C., Time-dependent ionisation of $\mathrm{H}$ and $\mathrm{He}$ in the local interstellar medium ApJ, 459, 216L-225L, 1996.

Malina, R.F., et al., Extreme ultraviolet explorer bright source list, AJ, 107, 751-764, 1994.

Margon B., et al., Extreme-ultraviolet observations of dwarf novae from Apollo-Soyuz ApJ, 224, 167-170, 1978.

Margon B., et al., An intense extreme-ultraviolet source in Cetus ApJ, 210, L79-L82, 1976.

Pounds, K.A., et al., The ROSAT wide field camera all-sky survey of extremeultravilet sources. I- The bright source catalogue MNRAS, 260, 77-102, 1993.

Priedhorsky, W.C., et al., ALEXIS-an ultrasoft X-ray monitor equipment using miniature satellite technology SPIE, 982, 188-207, 1988.

Pye, J.P., et al., The ROSAT wide field camera all-sky survey of extremeultravilet sources. II - The 2RE source catalogue MNRAS, 274, 1165-1193, 1995.

Sims, M.R., et al XUV wide field camera for Rosat OptEn, 29, 649-657, 1990.

Vennes, S., et al., The first detection of ionised helium in the ISM-EUVE and IUE spectroscopy of the hot white dwarf GD246 ApJ, 410, L119-L122, 1993 